\title{
A COMPARISON OF WAVELET AND S-TRANSFORM: FEATURE EXTRACTION AND CLASSIFICATION IN POWER QUALITY DATA MINING
}

\author{
Krishna B.V. \\ Saranathan College of Engineering, Trichy \\ E-mail: padhukrishna@ rediffmail.com
}

\begin{abstract}
This paper addresses the subject of power quality data mining and the role of signal transforms played in the above knowledge discovery process. We explore the performance of both Discrete Wavelet Transform (DWT) and S-transform in the feature extraction and classification stages of this mining process In our work, wavelet transform and S-transform(ST) were applied to transient power system data and pertinent features were extracted to further train a Learning Vector Quantization network (LVQ) for power disturbance classification. It was found that the number of features and hence the size and the training time of the LVQ network were considerably reduced in case of ST features. Also, the classification accuracy of the LVQ classifier was increased in the case of time -domain featured disturbances, such as sags, swell, etc when trained with ST features. Moreover, unlike Wavelet transform -based recognition system which is highly sensitive to the presence of noise, in the case of S-transform based system results are found to be quite satisfactory up to a noise level of $3.5 \%$.
\end{abstract}

Keywords: Power quality, data .mining, wavelet, S-transform, feature extraction

\section{INTRODUCTION}

In recent years, concern over the quality of electric power has been increasing rapidly since poor electric quality causes many problems for the affected loads, such as malfunctions, instabilities, short lifetime and so on. Poor quality of electric power is normally caused by line disturbances such as sags, notches and glitches. In order to improve electric power quality the sources and causes of such disturbances must be known before appropriate mitigating actions can be taken. Therefore, it is necessary to monitor these disturbances by sampling the current and voltage waveforms. Since transient power disturbances occur in the order of microseconds, a single -captured event recorded using this type of monitoring systems produces megabytes of data. [1].Another important aspect of power disturbance signals is the fact that the information of interest is often a combination of features that are well localized temporally or spatially (e.g. transients in power systems) [2]. The recorded data at a customer site could run into gigabytes in a year and this poses a great challenge for data storage, analysis and identification of disturbance data [3]. Thus raw transient power signal data could be analyzed using data mining techniques to provide knowledge about the captured waveforms and this is called as Power Quality Data Mining. Data Mining or knowledge discovery in databases $(K D D)$ is a process of automatic extraction of novel, useful and understandable patterns from a large collection of data. It can be viewed as a multidisciplinary activity because it exploits several research disciplines of artificial neural networks pattern recognition, expert systems, as well as mathematical disciplines such as statistics, information theory. A typical data mining process consists of the following steps data management, data preprocessing, data mining tasks algorithms and post processing. Many signal processing transforms like Fourier, Wavelet have been widely used to transform the data from one domain to the other and make them viable for classification. Wavelet theory could naturally play an important role in data mining since they have the properties of vanishing moments, hierarchical and multi-resolution decomposition structure, de-correlated coefficients etc. First wavelets could provide presentations of data that make the mining process more efficient and accurate. Secondly wavelets could be incorporated into the kernel of many data mining algorithms [4]. Wavelets have been used extensively for data management, preprocessing and data transformation. The S- transform is an extension to the ideas of wavelet transform, and is based on a moving and scalable localizing Gaussian window. The S-transform is unique in that it provides frequency -dependent resolution while it maintains a direct relationship with the Fourier spectrum [1]. S-transform has been used in the transformation stage of power quality data mining because it provides contours which 
closely resemble the disturbance patterns unlike the wavelet transform and hence the features extracted from it are very suitable for developing highly efficient and accurate classification.

Power quality data mining consists of two basic steps as given below

1. feature extraction

2. pattern recognition

In the feature extraction stage pertinent features suitable for further classification are extracted. In the second stage identification of power system patterns like sag, swell, transient is done. In our work initially wavelet transform was used to extract features from the raw power system data. Wavelet Multiresolution Analysis (MRA) is used along with Parseval's theorem to decompose the signal into thirteen levels and extract the corresponding energy disturbance feature at that level. These features were used to train a Learning Vector Quantization (LVQ) network. Secondly S-transform, a phase corrected Wavelet- transform has been used in the feature extraction stage. The number of features extracted and hence the size of the LVQ network and the training time are reduced. Also the classification accuracy was increased in the case of time- domain featured disturbances such as sag, swell etc. Moreover the performance of the system was found satisfactory up to a noise level of $3.5 \%$.

\section{DISCRETE WAVELET TRANSFORM}

\section{A. Wavelet Transform and Multiresolution Analysis Technique}

The Wavelet transform is a well-suited tool for analyzing the high frequency transients in the presence of low frequency components such as non-stationary and non-periodic wideband signals [11]. The first main characteristic in DWT is the MRA technique that can decompose the original signal into several other signals with different levels (scales) of resolution. From these decomposed signals, the original time-domain signal can be recovered without losing any information. The recursive mathematical representation of the MRA is as follows:

$\mathrm{V}_{j}=\mathrm{W}_{j+1} \oplus \mathrm{V}_{j+1}=\mathrm{W}_{j+1} \oplus \mathrm{W}_{j+2} \ldots \oplus \mathrm{W}_{j+n} \oplus \mathrm{V}_{\mathrm{m}+.}(1)$

\section{Where}

$\mathrm{V}_{j+1}$ approximated version of the given signal at scale $j+1$;
$\mathrm{W}_{j+1}$ detailed version that displays all transient phenomena of the given signal at scale $j+1$;

$\oplus \quad$ Denotes a summation of two decomposed signals;

$n \quad$ the decomposition level.

Fig. 1 illustrates the three decomposed /reconstructed levels of the DWT algorithm



Fig.1 Three decomposed/reconstructed levels of DWT.

At each decomposition level, the length of the decomposed signals (e.g., $u_{1}$ and $w_{1}$ ) is half that of the signals $\left(x_{0}\right)$ in the previous stage.

\section{B. Parseval's Theorem In DWT Application}

In Parseval's theorem, assuming a discrete signal $x[n]$ is the current that flows through the $1 \Omega$ resistance, then the consumptive energy of the resistance is equal to the squared sum of the spectrum coefficients of the Fourier transform in the frequency domain

$$
\frac{1}{N} \sum_{n=(N)}|x[n]|^{2}=\sum_{k=(N)}|a k|^{2}
$$

where $N$ is the sampling period, and $a_{k}$ is the spectrum coefficients of the Fourier transform.

To apply the theorem to the DWT, we use relevant mathematical equations for DWT and (2) to obtain (3) that is the Parseval's theorem in the DWT application

$$
\begin{gathered}
\left.\frac{1}{N}|\Sigma|_{t} X[f]\right|^{2}=\frac{1}{N_{j}} \Sigma\left|u_{j, k}\right|_{k}^{2} \\
+\frac{\Sigma}{N_{j}} 1_{j=1}\left(\Sigma\left|W_{j, k}\right|^{2}\right)
\end{gathered}
$$


Hence, through the DWT decomposition, the energy of the distorted signal is shown by (3). The first term on the right of (3) denotes the average power of the approximated version of the decomposed signal, while the second term denotes that of the detailed version of the decomposed signal. The second term giving the energy distribution features of the detailed version of distorted signal will be employed to extract the features of power disturbance.

\section{MULTIRESOLUTION S-TRANSFORM}

The Fourier transform of a time varying signal $h(t)$ is given by

$$
H(f)=\int_{-\infty}^{\infty} h(t) e^{i 2 \pi t t} d t
$$

The spectrum $H(f)$ is referred to as the "time-averaged spectrum." If the signal $h(t)$ is multiplied point by point with window function $g(t)$, then the resulting spectrum is

$$
H(f)=\int_{-\infty}^{\infty} h(t) g(t) e^{i 2 \pi f t} d t
$$

The S-transform is obtained by defining a particular window function in the form of a normalized Gaussian as

$$
g(t)=\frac{1}{\sqrt[\sigma]{2 \pi}} C^{-\left(t^{2} / 2 \sigma^{2}\right)}
$$

and then allowing the Gaussian to be a function of translation $\tau$ and dilation $\sigma$ (window width). The window width $\sigma$ is made proportional to the inverse of frequency and is chosen as

$$
O(f)=\frac{1}{a+b|f|}
$$

If $a=0, \sigma(f)$ denotes S-transform and for $b=0, \sigma(f)$ denotes a STFT. A typical value of $b$ varies between 0.333 to 5 , giving different frequency resolutions. For low frequencies, a higher value of $b$ is chosen and for high frequencies, lower value of $b$ is chosen to provide suitable frequency resolutions.

The FT of the window function is $g(t)$, obtained as

$$
G(u)=e^{2 \pi i^{2}}\left(\frac{1}{a+b \mid f}\right)^{2}
$$

S-transform produces a multiresolution analysis like a bank of filters with a constant relative bandwidth (constant analysis). The analysis window $w(t, f)$ is Gaussian and

$$
\int_{-\infty}^{\infty} g(t, f) d t=1
$$

Therefore

$$
\int_{-\infty}^{\infty} h(t) S(t, f) d r=H(f)
$$

Substituting (5) and (6) in (4), we get the

S-transform of $h(t)$ as

$$
S(r, f)=\int_{-\infty}^{\infty} h(t) g(r-f) e^{-R \pi t} d t
$$

Since $S(\tau, f)$ is complex, it can also be written as

$$
S(r, f)=A(r, f) e^{i p(r, f)}
$$

where $A(\tau, f)$ is amplitude S-spectrum and $\varphi(\tau, f)$ is the phase $\mathrm{S}$-spectrum. It can be noted that the S-transform improves the STFT in that it has a better resolution in phase space (i.e., a more narrow time window for higher frequencies) giving a fundamentally more sound time frequency representation. The discrete version of the S-transform is calculated by taking the advantage of the efficiency of the fast Fourier transform (FFT) and the convolution theorem. The discrete Fourier transform of the sampled signal $h(k T)$, $k=0,1, N-1$ is

$$
H\left[\frac{n}{N T}\right]=\frac{1}{N} \sum_{k=0}^{N-1} h(k T) e^{-i(2 \pi n K / N)}
$$

and discrete version of the S-transform of $h(k T)$ is obtained as (by letting $f \rightarrow n / N T$ and $\tau \rightarrow j T$ ) 


$$
S\left(j T, \frac{n}{N T}\right)=\int_{m=0}^{N-1} H\left[\frac{m+n}{N T}\right] G(m, n) e^{\frac{2 \pi n j}{N}}
$$

Where

$$
G(m n)=e-\left(2 \pi^{3} m^{2} \alpha^{2} / n^{2}\right)
$$

$\alpha=1 / b, j, m$, and $n=0,1,2, \ldots N-1$ and $N=$ total number of samples.

The discrete inverse of S-transform is obtained as given by (16). The various steps for computing S-transform are outlined as follows.

$$
h(k T)=\frac{1}{N} \sum_{n=0}^{N-1}\left\{\sum_{j=0}^{N-1} S\left[\frac{n}{N T} j T\right]\right\} e^{i(2 \pi n k / N)}
$$

1. Denote $n / N T, m / N T, k T$, and $j T$ as $n, m, k$, and $j$, respectively, for all of the computations.

2 Obtain discrete Fourier transform $H(m)$ of the original time-varying signal $\mathrm{h}(t)$, with $\mathrm{N}$ points and sample interval $T$, using FFT routine from (13).

3. Compute the localizing Gaussian $\mathrm{G}[n, m]$ for the required frequency using (15).

4. Shift the spectrum $H[m]$ to $H[m+n]$ for the frequency $n$ by using convolution theorem.

5. Determine $B(n, m)=H(m+n) \cdot G(m+n)$.

6. Compute inverse Fourier transform of $\mathrm{B}(n, m)$ from $m$ to j to give the row of $S[n, j]$ corresponding to the frequency $n$.

7. Repeat steps 3,4 , and 5 until all of the rows of $S[n, j]$ corresponding to all discrete frequencies have been obtained.

The total number of operations for computing S-transform is $N(N+N \log N)$.

The multiresolution S-transform output is a complex matrix, the rows of which are the frequencies and the columns are the time values. Each column thus represents the "local spectrum" for that point in time. Also, frequency-time contours having the same amplitude spectrum are obtained to detect, and localize power disturbance events.

\section{FEATURE EXTRACTION}

\section{A COMPARISON OF WAVELET AND S-TRANSFORM}

\section{A. Signal Modeling}

Signals were generated by using parametric equations as given in Table 1 and Table 2 .It was possible to change testing and training signal parameters in a wide range and in a controlled manner. These signals are also mixed with random white noise of zero mean. Ten cycles of power frequency $(50 \mathrm{~Hz}$, amplitude 1p.u.) were taken under consideration and sampled at the rate of $15.2 \mathrm{Khz}$. The disturbances namely sag, swell, harmonics, transients were simulated using matlab.

\begin{tabular}{|c|c|}
\hline Event & Equation \\
\hline Pure sine & $v(t)=\sin (w t)$ \\
\hline Sudden Sag & $v(t)=(1-s s((t-t 2)-(t-t 1))) \sin (w t)$ \\
\hline Sudden Swell & $v(t)=(1+\operatorname{sw}((t-t 2)-(t-t 1))) \sin (w t)$ \\
\hline Harmonics & $\begin{array}{l}v(t)=(1 \\
\sin (w t)+2 \sin (3 w t)+3 \sin (5 w t)+\ldots \ldots \ldots)\end{array}$ \\
\hline Transient & $\begin{array}{l}V(t)=(\sin (w t)+s s \exp (-(t-t b) /) o s c) \\
\sin (w(t-t b))\end{array}$ \\
\hline
\end{tabular}

\section{Table I. Parametric Eqns for simulation of distorted signals}

Table II. Parametric variations of simulated signals

\begin{tabular}{|l|l|}
\hline \multicolumn{1}{|c|}{ Event } & \multicolumn{1}{|c|}{ Parametric variation } \\
\hline Pure sine & Amplitude 1,frequency $50 \mathrm{~Hz}$ \\
\hline Sudden Sag & $\begin{array}{l}\text { Duration }(\mathrm{t} 2-\mathrm{t} 1)=(0-9) T \\
\text { Amplitude } \mathrm{ss}=0.3-0.8\end{array}$ \\
\hline Sudden Swell & $\begin{array}{l}\text { Duration }(\mathrm{t} 2-\mathrm{t} 1)=(0-9) T \\
\text { Amplitude } \mathrm{ss}=0.3-0.8\end{array}$ \\
\hline Harmonics & $\begin{array}{l}\text { Order } 3,5,7 \text { amplitudes } 0-.9 \\
\text { frequency=100-400 } \mathrm{Hz}\end{array}$ \\
\hline Transient & $\begin{array}{l}\text { Time const: } 0.008-0.04 \mathrm{~s} \\
\text { frequency=100-400 } \mathrm{Hz}\end{array}$ \\
\hline
\end{tabular}




\section{B. Feature Extraction using DWT}

\section{Energy distribution feature}

Wavelet MRA along with Parseval's theorem was employed to decompose the original signal into thirteen levels and extract the corresponding detailed energy concentration at that level. Thirteen features were extracted for each of the thirteen levels along with the disturbance duration (total of 14 features). These fourteen features were given as inputs to a LVQ network Fig 2 shows the energy distribution for a swell disturbance. Ten cycles of power frequency were considered of which three cycles had a swell of 0.8 p.u. Figs 3, 4, 5, 6 show sag, outage, harmonics, transient signals and their respective energy distribution diagram.

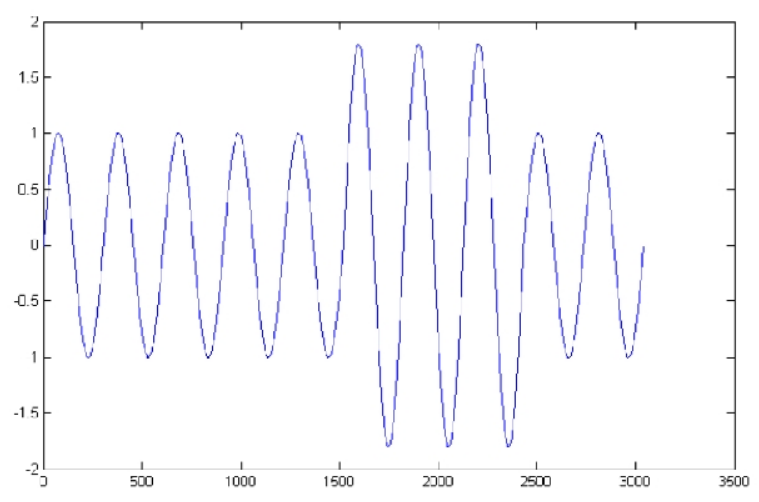

(a)

Fig. 2 (a) Voltage swell (b) corresponding energy

\section{Duration of Electromagnetic Transient:}

In general, when a transient disturbance occurs, the stable power signal will generate a discontinuous state at the start and end points of the disturbance duration. Employing the DWT technique to analyze the distorted signal through one-level decomposition of the MRA will cause the wavelet coefficients $w_{1}$ at the start and end points of the disturbance to generate severe distribution variation. Therefore, we can easily obtain the start time $t_{\mathrm{s}}$ and end time $t_{\mathrm{e}}$ of the disturbance duration from the variations in absolute wavelet coefficients $w_{1}$ and calculate the disturbance duration $t_{\mathrm{t}}$

$$
t_{t}=\left|t_{e}-t_{s}\right|
$$

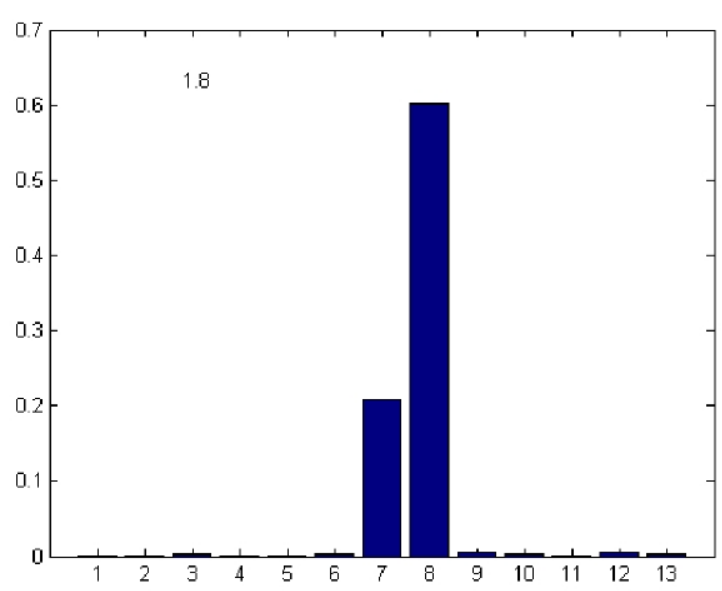

(b)

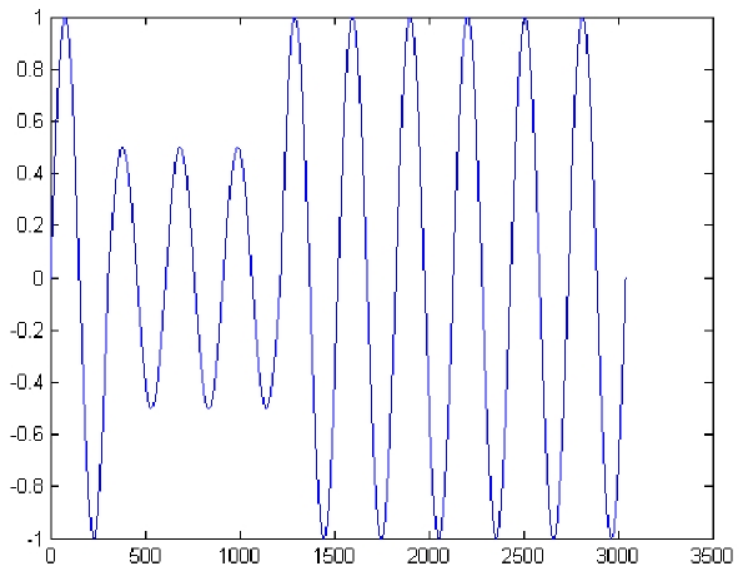

(a)

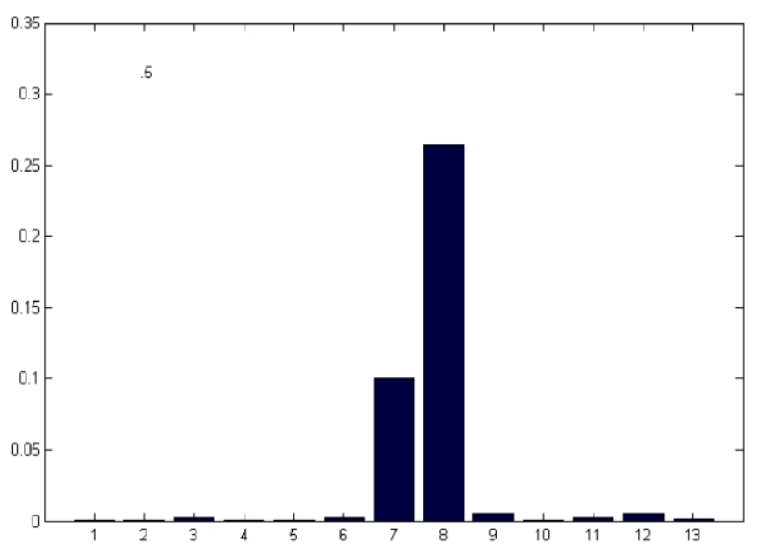

(b)

Fig. 3 (a) Voltage sag (b) corresponding energy 


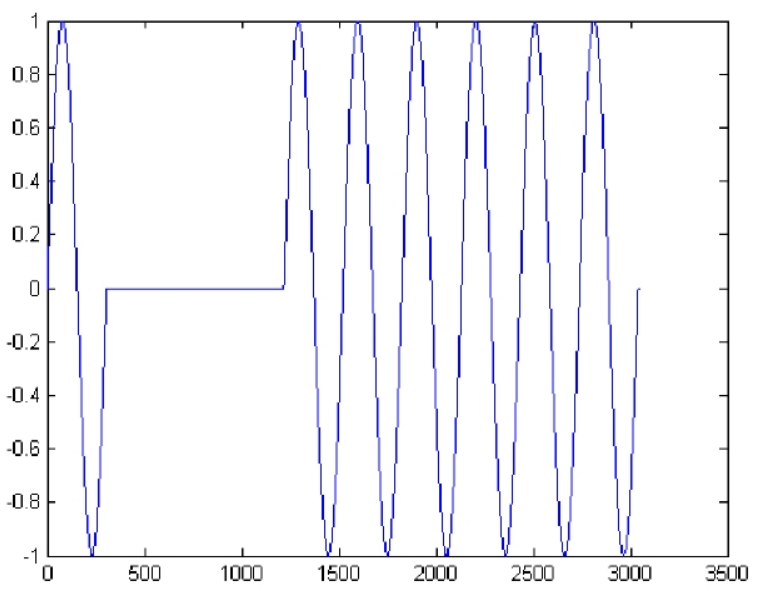

(a)

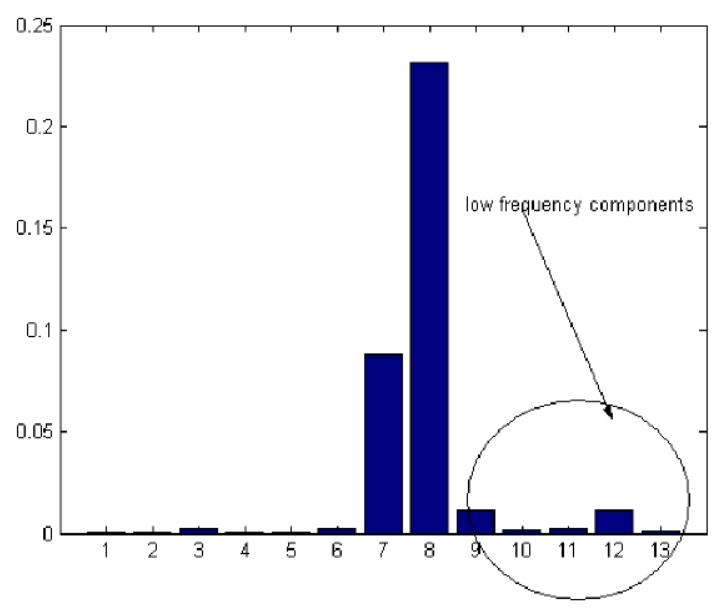

(b)

Fig. 4 (a) Voltage outtage (b) corresponding energy



(a)

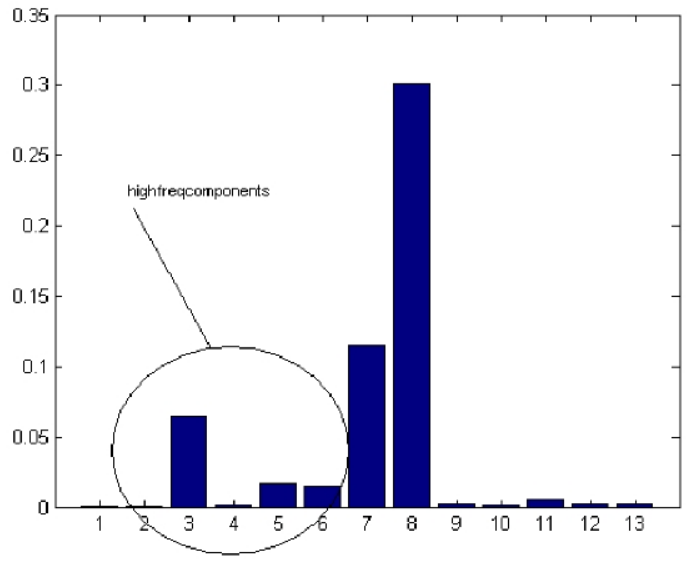

(b)

Fig. 5 (a) Harmonics (b) corresponding energy distribution

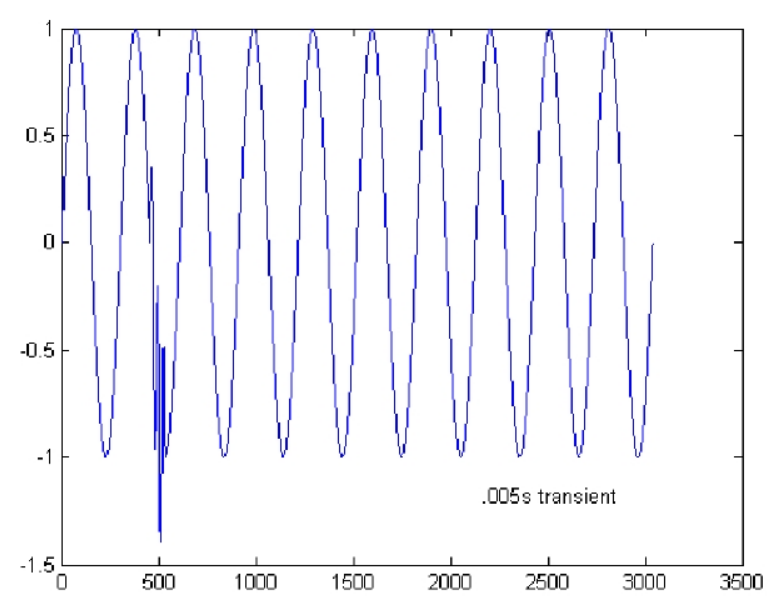

(a)

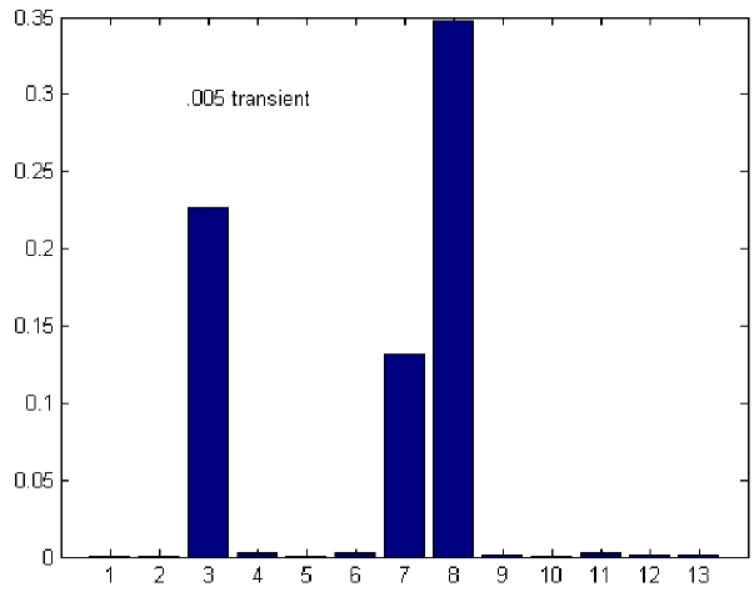

(b)

Fig. 6 (a) Transients (b) corresponding energy distribution 


\section{Feature extraction using S-transform}

The S-transform yields contours which are very much viable to visual inspection. An example of 3 cycle sag disturbance has been considered. Fig 7 (a) shows the disturbance signal (b) amplitude Vs time (c) amplitude Vs normalized frequency. Fig 7 (b) has been obtained by searching rows of S-transform matrix. There is a reduction in magnitude during the three cycles of sag. Similarly Fig. 7 (c) gives the maximum frequency content of the voltage signal. Fig 8 shows the time-frequency contours. Visual inspection of the contours shows that the curves follow the disturbance pattern. Fig 9,10,11,12 represent similar curves for swell, harmonics signals.

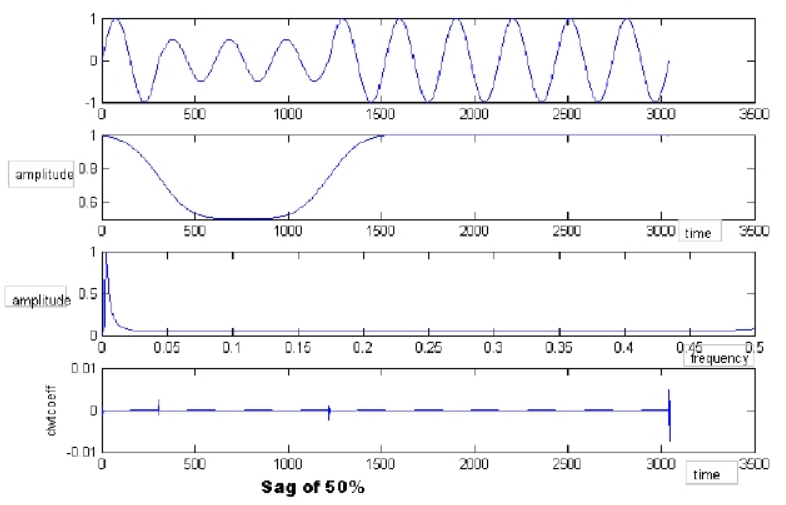

Fig. 7. S-transform plots for sag (a) signal (b) amplitude Vs time (c) amplitude Vs freq (d) dwt coeff

The features that were extracted by applying S-transform are as follows

1. The standard deviation (std1) of the lowest-transform contour that is above the normalized fundamental frequency.

2. The standard deviation(std2) of the amplitude Vs time curve obtained from, the rows of the S-transform matrix that is above two times the normalized frequency.

3. $a m p=\max (\mathrm{Sa})+\min (\mathrm{Sa})-\max (\mathrm{Sb})-\min (\mathrm{Sb})$

where Sa denotes the rows of the S-matrix output with disturbance, and $\mathrm{Sb}$ denotes the corresponding output without disturbance. It is to be noted that for the window factor (alpha) is set as alpha $=2$ for sag, swell and harmonics and pure sine whereas for transients alpha is taken as 3 . The number of features extracted were three along with duration of disturbance

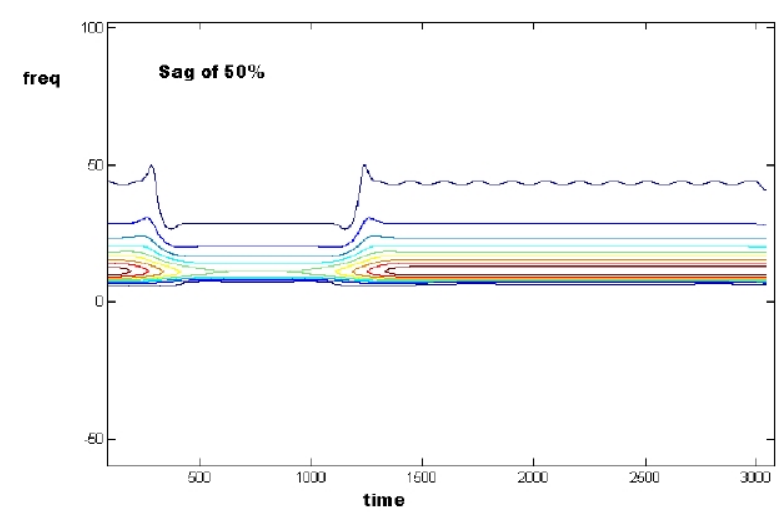

Fig. 8. S-transform contours for sag Freq Vs time



Fig. 9. S-transform plots for swell (a) Signal (b) amplitude Vs time (c) amplitude Vs freq

tt (4 features). The number of features is four when compared to fourteen features in wavelet transform. This reduction in number of features leads to reduction in size of the neural classifiers.

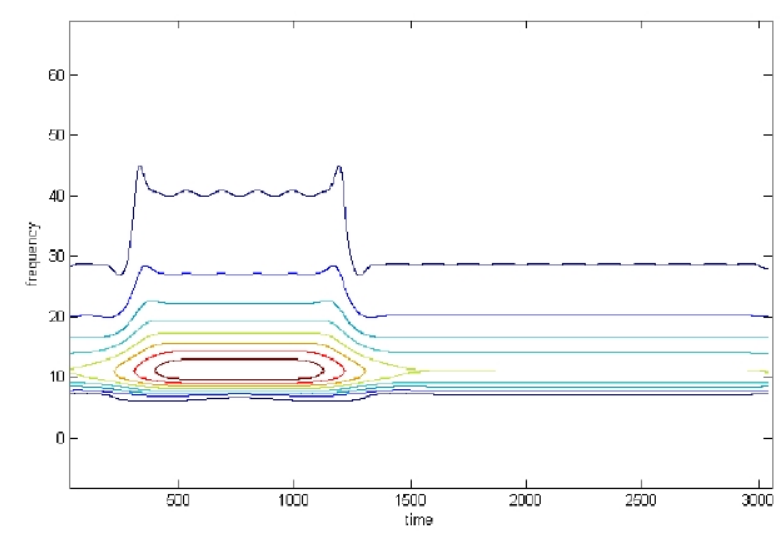

Fig. 10 S-transform contours for swell Freq Vs time 


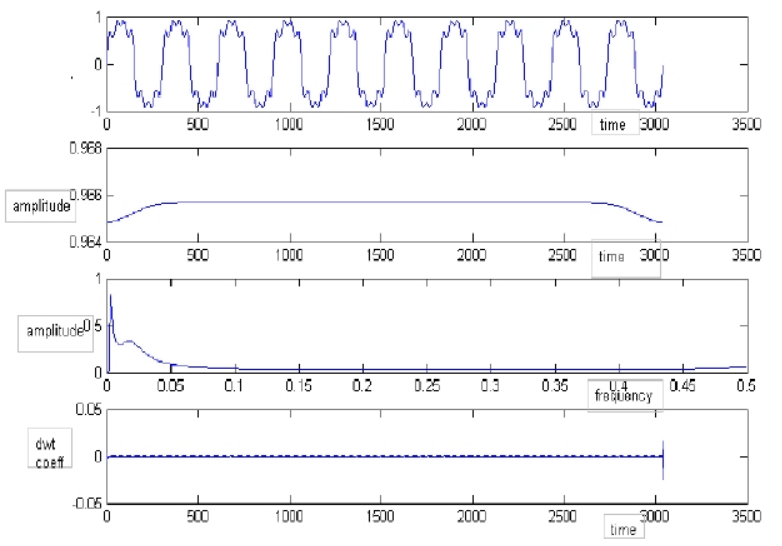

Fig. 11. S-transform plots for harmonics 3, 5, 7 (a) signal (b) amplitude Vs time (c) amplitude Vs freq (d) dwt coeff

\section{CLASSIFICATION RESULTS USING LEARNING VECTORNETWORK (LVQ)}

\section{A. Wavelet based LVQ}

Five categories of power system disturbances were simulated, namely swel I(S1), sag (S2), harmonics (S3) transient1 (S4), transient 2 (S5).Transient 1 has a spectral component of $500 \mathrm{~Hz}$ and duration of .005 sec while transient 2 has a duration of. $01 \mathrm{sec}$. Pure sine wave is considered to be class zero. For each of the five classes, 50 different signals were generated accounting for a total of 250 . The signal parameters were changed as given in the Table I and Tablell. 14 features were extracted by applying wavelet MRA and Parseval's theorem for each disturbance signal. Table III $A$ and $B$ show features extracted by DWT while Table IV show that extracted by S-transform. 25 signals of each class were used for training and the rest for testing network with and without noise. Table V,VI, give

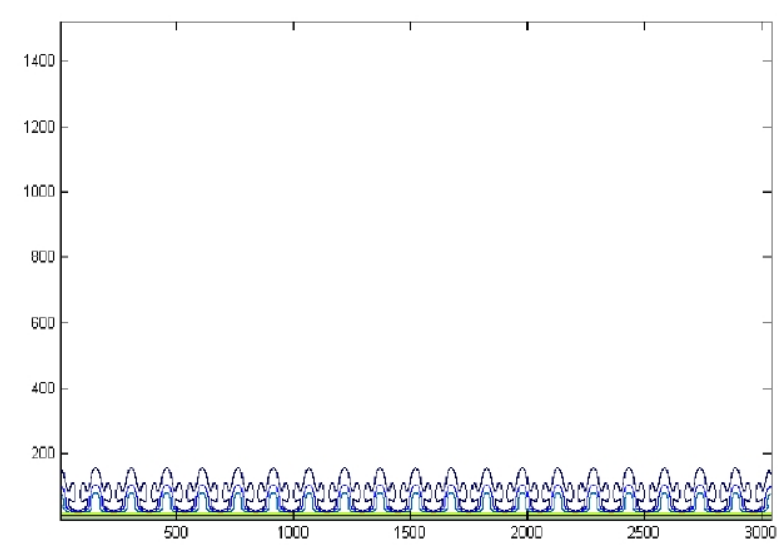

Fig. 12. S-transform contours for harmonics Freq Vs time

the classification results of wavelet based LVQ network with and without noise. Every row gives the information as to how many signals belonging to a class were actually classified or misclassified. The classification results were not very accurate in case of transients, sags, swells. Also accuracy dropped with noise.

\section{B. S-transform based LVQ}

The S-transform based classifier showed better results than the wavelet classifier for both without and with the presence of noise. Table VII, VIII, give the classification results of S-transform based LVQ network with and without Also it shows better classification accuracy in case of transients, sag and swells.

\section{CONCLUSION}

In this paper, a comparative study of wavelet and S-transform in the feature extraction stage and classification stage has been done. The wavelet transform is better than the Fourier transform; yet it

Table III. Features Extracted By Dwt

A

\begin{tabular}{|l|c|c|c|c|c|c|c|c|}
\hline \multicolumn{1}{|c|}{ signal type } & $\begin{array}{c}\text { energy } \\
\text { level1 }\end{array}$ & $\begin{array}{c}\text { energy } \\
\text { level2 }\end{array}$ & $\begin{array}{c}\text { energy } \\
\text { level3 }\end{array}$ & $\begin{array}{c}\text { energy } \\
\text { level4 }\end{array}$ & $\begin{array}{c}\text { energy } \\
\text { level5 }\end{array}$ & $\begin{array}{c}\text { energy } \\
\text { level6 }\end{array}$ & $\begin{array}{c}\text { energy } \\
\text { level7 }\end{array}$ & Class \\
\hline swell & $2.46 \mathrm{E}-08$ & $3.51 \mathrm{E}-07$ & 0.002 & $1.25 \mathrm{E}-05$ & $8.11 \mathrm{E}-05$ & 0.0035 & 0.2205 & 1 \\
\hline sag & $1.72 \mathrm{E}-08$ & $2.86 \mathrm{E}-07$ & 0.0019 & $8.70 \mathrm{E}-06$ & $5.33 \mathrm{E}-05$ & 0.002 & 0.1007 & 2 \\
\hline harmonics & $1.28 \mathrm{E}-07$ & $2.62 \mathrm{E}-06$ & 0.065 & 0.00169 & 0.01661 & 0.0151 & 0.1147 & 3 \\
\hline Transient .005sec & $4.98 \mathrm{E}-07$ & $5.76 \mathrm{E}-06$ & 0.22 & 0.00206 & 0.00086 & 0.0023 & 0.1315 & 4 \\
\hline Transient .01sec & $4.99 \mathrm{E}-07$ & $6.07 \mathrm{E}-06$ & 0.3 & 0.00272 & 0.0033 & 0.0022 & 0.1311 & 5 \\
\hline
\end{tabular}


B

\begin{tabular}{|l|c|c|c|c|c|c|c|c|}
\hline \multicolumn{1}{|c|}{ signal type } & $\begin{array}{c}\text { energy } \\
\text { level8 }\end{array}$ & $\begin{array}{c}\text { energy } \\
\text { level9 }\end{array}$ & $\begin{array}{c}\text { energy } \\
\text { level10 }\end{array}$ & $\begin{array}{c}\text { energy } \\
\text { level11 }\end{array}$ & $\begin{array}{c}\text { energy } \\
\text { level12 }\end{array}$ & $\begin{array}{c}\text { energy } \\
\text { level13 }\end{array}$ & tt & Class \\
\hline swell & 0.59009 & 0.00061 & 0.0013 & 0.0052 & 0.0012 & 0.0017 & 0.06 & 1 \\
\hline sag & 0.2641 & 0.00533 & 0.0008 & 0.0021 & 0.0007 & 0.0011 & 0.06 & 2 \\
\hline harmonics & 0.30155 & 0.00207 & 0.0014 & 0.0058 & 0.0016 & 0.0024 & 0.0001 & 3 \\
\hline Transient .005sec & 0.34717 & 0.0015 & 0.0006 & 0.0025 & 0.0009 & 0.0015 & 0.005 & 4 \\
\hline Transient .01sec & 0.34949 & 0.00137 & 0.0007 & 0.0025 & 0.0009 & 0.0014 & 0.01 & 5 \\
\hline
\end{tabular}

Table IV. Features Extracted By S-Transform

\begin{tabular}{|l|c|c|c|c|c|c|}
\hline \multicolumn{1}{|c|}{ Type } & std1 & std2 & amplitude & tt & class & window \\
\hline swell $90 \%$ & 0.1771 & 0.0838 & 0.8989 & 0.06 & 1 & alpha $=0.2$ \\
\hline swell $10 \%$ & 0.0197 & 0.0094 & 0.0988 & 0.06 & 1 & alpha $=0.2$ \\
\hline sag $90 \%$ & 0.0195 & 0.0091 & -0.0999 & 0.06 & 2 & alpha $=0.2$ \\
\hline sag 10\% & 0.1768 & 0.0811 & -0.8994 & 0.06 & 2 & alpha $=0.2$ \\
\hline Harmonics & 0.0003 & 0.014 & -0.0347 & 0.01 & 3 & alpha $=0.2$ \\
\hline transient(.01sec) & 0.0045 & 0.126 & 0.1386 & 0.0001 & 4 & alpha $=3$ \\
\hline transient1(.005sec) & 0.0002 & 0.0523 & 0.001 & 0.005 & 5 & alpha $=3$ \\
\hline pure sine & $1 \mathrm{E}-06$ & $1 \mathrm{E}-06$ & $1 \mathrm{E}-06$ & 0.2 & 0 & alpha $=0.2$ \\
\hline
\end{tabular}

Table V. Wavelet - transform based LVQ without noise

\begin{tabular}{|l|c|c|c|c|c|c|}
\hline & swell S1 & sag S2 & harmonics S3 & transient1S4 & transient2 S5 & puresine S0 \\
\hline swell S1 & 22 & & & & & 3 \\
\hline sag S2 & & 22 & & & & 3 \\
\hline harmonics S3 & & & 24 & & & 1 \\
\hline transient1 S4 & & & & 21 & 2 & 2 \\
\hline transient2 S5 & & & & 2 & 21 & 2 \\
\hline
\end{tabular}


Table VI. Wavelet - transform based LVQ with noise of $3.5 \%$

\begin{tabular}{|l|c|c|c|c|c|c|}
\hline & swell S1 & sag S2 & harmonics S3 & transient1S4 & transient2 S5 & puresine S0 \\
\hline swell S1 & 21 & & & & & 4 \\
\hline sag S2 & & 21 & & & & 4 \\
\hline harmonics S3 & & & 23 & & & 2 \\
\hline transient1 S4 & & & & 20 & 2 & 3 \\
\hline transient2 S5 & & & & 2 & 20 & 3 \\
\hline
\end{tabular}

Classification accuracy $=92 \%$

Table VII. S - transform based LVQ without noise

\begin{tabular}{|l|c|c|c|c|c|c|}
\hline & swell S1 & sag S2 & harmonics S3 & transient1S4 & transient2 S5 & puresine S0 \\
\hline swell S1 & 24 & & & & & 1 \\
\hline sag S2 & & 24 & & & & 1 \\
\hline harmonics S3 & & & 24 & & & 1 \\
\hline transient1 S4 & & & & 23 & 1 & 1 \\
\hline transient2 S5 & & & & 1 & 23 & 1 \\
\hline
\end{tabular}

Classification accuracy $=97.2 \%$

Table VIII. S - transform based LVQ with noise of $3.5 \%$

\begin{tabular}{|l|c|c|c|c|c|c|}
\hline & swell S1 & sag S2 & harmonics S3 & transient1S4 & transient2 S5 & puresine S0 \\
\hline swell S1 & 23 & & & & & 2 \\
\hline sag S2 & & 23 & & & & 2 \\
\hline harmonics S3 & & & 24 & & & 1 \\
\hline transient1 S4 & & & & 22 & 2 & 1 \\
\hline transient2 S5 & & & & 2 & 22 & 1 \\
\hline
\end{tabular}

Classification accuracy $=95.6 \%$

requires a large number of neural networks. Moreover the classification accuracy of a wavelet-based recognition system may be limited if an important disturbance frequency component has not been precisely extracted by the wavelet MRA. Also its performance in presence of noise is unsatisfactory. The S-transform with a variable window is used to generate contours and feature vectors that reduce the network size and training time of the neural classifiers. The proposed S-transform based LVQ network uses only four features when compared to 14 features in the wavelet - based system. This increases the computational accuracy and reduces memory space. Moreover S-transform has more noise immunity than DWT based classifiers. The average classification accuracy of S-transform based LVQ network is $96.4 \%$ whereas its wavelet counterpart has an accuracy of $93 \%$

\section{REFERENCES}

[1] P.K.Dash, B.K.Panigrahi, D.K.Sahoo and G.Panda, "Power quality disturbance data compression, detection and classification using integrated spline and S-transform", IEEE Trans. Power Delivery, vol. 18, No 2, Apr. 2002, pp.595-600 
[2] Cheng-Hsing Lee, Yaw JUEn Wang and Wen Llang Huang, "A literature survey of wavelets in power engineering applications", in 1987 Proc. National Science Council, ROC(A),24(4),pp. 249-25

[3] P.K.Dash, lan Lee Wen Chung, M.V.Chilukuri," Power quality data mining using soft-computing and wavelet transform", TENCON 2003, Conference on Convergent Technologies for the Asia-Pacific Region, vol.3, pp.976-980

[4] Tao LI ,Qi Li,Shenghuo Zhu,Mitsunori Ogihara," A survey of wavelet applications in data mining", SIGKDD Explorations ,Vol 4,No 2,pp 49-69

[5] W.Kanitpanyacharocan and S. Premrude eprecharan, "Power Quality problem classification using Wavelet transformation and Neural Networks," IEEE Power Systems Conference and Exposition, vol13, pp.1496-1500, 10-13 Oct 2004.

[6] T.K.AbdelGalil, M.Kamel, A.M. Youssef, E.F.Youssef and E.F.Saadany, "Power quality disturbance classification using the inductive inference approach," IEEE Trans. Power Delivery,vol 19, no 4,pp.1812-1818,Oct 2004

[7] D.C.Robertson,L.Octavia and J.Mayu, "Wavelet and power system transient feature extraction and classification", in SPIE Proc. Signal and Image Processing ,1994,vol 2242,pp 478-487

[8] A.K.Gosh and D.L.Lubkeman, "The classification of power system disturbance waveforms using a neural network approach," ",IEEE Trans. Power Delivery, vol 10,no 1,pp.109-115,Jan 1995.

[9] Surya Santoso, Edward J.Powers, W.MackGrady, "Power Quality Disturbance Waveform -Based Neural
Classifier- II Application", IEEE Trans. Power Delivery, vol 15,no 1,pp.229-235, Jan2000.

[10] Jachak Chung, E. J. Powers,W. M. Grady, and S.C.Bhatt, "Power disturbance classifier using a Rule-based method and Wavelet Packet -Based hidden Markov model", IEEE Trans. Power Delivery, vol. 17, no 1, Jan. 2002.

[11] S. Santoso, E. J. Powers,W. M. Grady, and P. Hofmann, "Power quality assessment via wavelet transform analysis," IEEE Trans. Power Delivery, vol. 11, no. 2,pp. 924-930, Apr. 1996.

[12] T. Kohonen, "The self-organizing map," Proceedings of the IEEE, vol.78, no. 9, pp. 1464-1480, Sept. 1990.

[13] T. Kohonen, "Self-Organizing Maps."Heidelberg: Springer, 1995.

[14] S. Haykin, "Neural Networks, A Comprehensive Foundation." New York, NY: Macmillan College Publishing Company, 1994.

[15] Surya Santoso, Edward J.Powers, W. MackGrady, "Power Quality Disturbance Waveform -Based Neural Classifier-Part1 Theoretical Foundation", IEEE Trans.Power Delivery,vol 15,no1,pp.222-228, Jan 2000

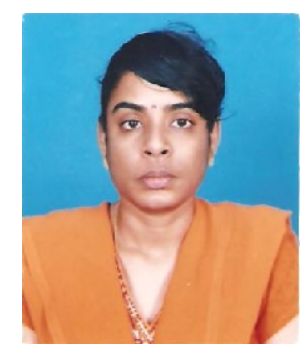

B.V.Krishna is now pursuing $\mathrm{PhD}$ in data-mining from Anna University, India. Her areas of interest includes signal processing, artificial intelligence and machine learning algorithms 